\title{
Tumor germinal mixto con componentes de disgerminoma y coriocarcinoma de ovario en mujer adolescente con ataxia- telangiectasia
}

\begin{abstract}
RESUMEN
Antecedentes: la ataxia-telangiectasia es una enfermedad hereditaria con patrón de herencia autosómico recesivo. Se caracteriza por deterioro neurológico, telangiectasias e inmunodeficiencia. Su causa es una mutación en el gen ATM (ataxia telangiectasia mutated) localizado en el cromosoma 11q22.3-23.1, relacionado con la síntesis de una proteína con actividad fosfoinositol-3-cinasa que participa en la regulación del ciclo celular y en la reparación del ácido desoxirribonucleico; esto explica, en parte, la susceptibilidad al desarrollo de neoplasias. Su asociación con algunos tipos de cáncer se ha establecido previamente y la mayor incidencia es con leucemias y linfomas. Se han reportado siete niñas con ataxia-telangiectasia asociada con tumores sólidos germinales, específicamente con disgerminoma.
\end{abstract}

Caso clínico: mujer adolescente de 12 años con diagnóstico de ataxiatelangiectasia desde los tres años. Tuvo infecciones repetidas de las vías respiratorias que requirieron múltiples hospitalizaciones de dos a tres veces por mes; recibió tratamiento con inmunoglobulina y profilaxis antimicrobiana. A los 12 años acudió al servicio de urgencias con abdomen agudo; fue operada y se halló una masa abdominopélvica que se extirpó. El diagnóstico fue de tumor germinal mixto con componente de disgerminoma y coriocarcinoma de ovario. Recibió un primer ciclo de quimioterapia con bleomicina $\left(10 \mathrm{UI} / \mathrm{m}^{2} /\right.$ día, una dosis), ciclofosfamida ( $1 \mathrm{~g} / \mathrm{m}^{2} /$ día por cuatro días) y cisplatino $\left(20 \mathrm{mg} / \mathrm{m}^{2} /\right.$ dosis por cinco días). Se suspendió la quimioterapia porque desarrolló choque séptico que puso en riesgo su vida. Por su enfermedad de base permaneció bajo vigilancia. Actualmente, 17 meses después, la paciente vive sin actividad tumoral.

Conclusión: existe asociación entre ataxia-telangiectasia, leucemia y linfomas. Se describe el caso clínico de una paciente con ataxia-telangiectasia y tumor germinal mixto con componente de disgerminoma y coriocarcinoma de ovario. Se propone establecer un tratamiento especial para estos pacientes inmunocomprometidos, con alto riesgo de cáncer pero, a la vez, de no tolerar los esquemas habituales.

Palabras clave: tumor germinal de ovario, disgerminoma, coriocarcinoma, adolescente, ataxia-telangiectasia.
Eduardo Augusto Gálvez-Cuitiva ${ }^{1}$

Cecilia Ridaura-Sanz ${ }^{2}$

Marco Antonio Yamazaki-Nakashimada ${ }^{3}$

Carlos Leal-Leal ${ }^{4}$

Marta Zapata-Tarrés ${ }^{4}$

${ }^{1}$ Pediatría.

${ }^{2}$ Departamento de Patología.

${ }^{3}$ Servicio de Inmunología.

${ }^{4}$ Servicio de Oncología.

Instituto Nacional de Pediatría, México.

Recibido: 5 de febrero del 2014

Aceptado: 20 de abril del 2015

Correspondencia: Marta Zapata Tarrés Servicio de Oncología Instituto Nacional de Pediatría Insurgentes Sur 3700-C CP 04530 México, D.F. Tel: 5215554184099

magazapata@yahoo.com

Este artículo debe citarse como Gálvez-Cuitiva EA, Ridaura-Sanz C, YamazakiNakashimada MA, Leal-Leal C, Zapata-Tarrés M. Tumor germinal mixto con componentes de disgerminoma y coriocarcinoma de ovario en mujer adolescente con ataxia-telangiectasia. Acta Pediatr Mex 2015;36:464-472. 


\title{
Germ cell ovarian tumor in an adolescent with ataxia-telangiectasia
}

\begin{abstract}
Background: Ataxia-telangiectasia (AT) is an autosomal recessive hereditary disease characterized by neurological deterioration, telangiectasias and immunodeficiency. The cause is a punctual mutation in ATM gene localized in chromosome 11q22.3-23.1, which translates into a phosphoinositol 3-kinase protein. This protein regulates the cell cycle and the repair of the DNA. This defect partially explains the increased risk of cancer. The association of the AT with cancer has been already established, being the major incidence of leukemias and lymphomas. In the literature, seven girls have been reported with the association of AT and germ cell solid tumors, specifically with dysgerminoma.
\end{abstract}

Case report: We present the case of a 12-year-old female who was diagnosed with AT since 3 years of age. She presented recurrent respiratory tract infections requiring multiple hospitalizations and was treated with immunoglobulin and antimicrobial prophylaxis. She was admitted in the emergency ward with acute abdomen and was evaluated by surgical oncology and operated. An abdominal mass was found and resected. Pathology reported an ovaric mixed germ cell tumor with coriocarcinoma and dysgerminoma. She was treated with one dose of bleomicin $\left(10 \mathrm{UI} / \mathrm{m}^{2}\right)$, cyclophosphamide $\left(1 \mathrm{~g} / \mathrm{m}^{2} /\right.$ day for four days) and cysplatin (20 mg/m²/day for five days). During chemotherapy she presented a live threatening septic shock. Because of AT it was decided to stop chemotherapy. At present, the patient is alive without tumor activity for 17 months.

Conclusion: AT is associated with leukemias and lymphomas. We report a case of an AT patient with ovarian tumor, coriocarcinoma and dysgerminoma components. A special approach is proposed for these inmunocompromised patients who are leaving more and are at high risk of cancer but may not tolerate standard treatments.

Key word: Cancer of ovary, dysgerminoma, choriocarcinoma, adolescent, ataxia telangiectasia.

\section{INTRODUCCIÓN}

La asociación entre enfermedades o síndromes genéticos y neoplasias malignas permite identificar alteraciones genéticas que pudieran relacionarse con la fisiopatología del cáncer. Por esta razón presentamos el caso de una mujer adolescente con ataxia-telangiectasia y tumor germinal mixto con componentes de disgerminoma y de coriocarcinoma de ovario.

La ataxia-telangiectasia, o síndrome de Louis Bar, se describió en 1926 por Sillaba y Hennery y por Louis Bar en 1941..$^{1,2}$ Hasta 1957 
Boder y Sedgick le dieron el nombre de ataxia-telangiectasia. ${ }^{3}$ Ésta es una enfermedad neurodegenerativa, de origen autosómico recesivo, causada por mutaciones del gen ataxia-telangiectasia mutated (ATM) localizado en el cromosoma 11 (11q22-23)..$^{1-3}$ Esta proteína normalmente reconoce rupturas del ADN y activa mecanismos de reparación.

Las mutaciones en el gen son causas de la ausencia de la proteína o de su función, ya que media sus funciones a través de su interacción con otras proteínas como la Pin2 que regula los factores "Gap 2 (intervalo 2)" (G2) y de la mitosis (M) del ciclo celular; la proteína TR F1 (factor de unión a repeticiones teloméricas 1 ), que regula negativamente la elongación telomérica e induce la entrada a las fases de mitosis y apoptosis; ${ }^{4}$ la ligasa 4, que actúa sobre el crecimiento de los fibroblastos embriónicos y sobre la inestabilidad genómica, ${ }^{5}$ además de fosforilar otras proteínas como la BRCA 1 (cáncer de mama 1, breast cancer 1) relacionada con el cáncer de mama y el F-4E (factor 4E de iniciación de la traducción eucariótica) relacionado con la resistencia a la insulina. ${ }^{5,6}$

La asociación entre la ataxia-telangiectasia y el cáncer ha sido establecida previamente; la mayor incidencia es con leucemias y linfomas. En este caso, se ratifica la asociación con tumores sólidos germinales, específicamente con el cáncer de ovario. Por otro lado, los tumores germinales no suelen asociarse con ningún síndrome genético o estado clínico específico y suelen presentarse en pacientes previamente sanos. El disgerminoma de ovario es un tumor germinal que ocurre con mayor frecuencia en mujeres jóvenes o adolescentes, su cuadro clínico clásico es el de un abdomen agudo y frecuentemente es unilateral; la variedad que contiene coriocarcinoma se caracteriza por una presentación clínica más agresiva sumada a pubertad precoz en algunos casos.

\section{CASO CLÍNICO}

Mujer de 12 años de edad cuyo padecimiento inició al cumplir un año con marcha atáxica y nistagmo horizontal bilateral. A los tres años se evidenciaron telangiectasias en piel y en las conjuntivas oculares. Con base en dicho cuadro clínico y por el deterioro progresivo se diagnosticó ataxia-telangiectasia (Figura 1). La paciente tuvo infecciones repetidas de vías respiratorias altas y bajas que requirieron manejo hospitalario en varias ocasiones. Dos de ellas se trataron en la unidad de terapia intensiva por neumonía. Se le dio tratamiento con inmunoglobulina humana intravenosa mensual, inicialmente de lgG 610 mg/dL (normal para la edad 633-1 280 mg/ $\mathrm{dL}$ ), IgA $66 \mathrm{mg} / \mathrm{dL}$ (normal para la edad 33$206 \mathrm{mg} / \mathrm{dL}$ ), lgM $320 \mathrm{mg} / \mathrm{dL}$ (normal para la edad $48-207 \mathrm{mg} / \mathrm{dL}$ ) y profilaxis antimicrobiana con trimetoprim con sulfametoxazol (5 mg/mg/día). Se encontraba en seguimiento multidisciplinario por los servicios de oncología médica pediátrica, inmunología pediátrica y genética médica.

La vigilancia de estas neoplasias se realizó en citas semestrales a oncología con biometría hemática, ultrasonido abominal y alfafetoproteína sérica. El último valor de alfafetoproteína fue de $117 \mathrm{UI} / \mathrm{mL}$, cuatro meses antes del padecimiento actual que inició dos días antes de su

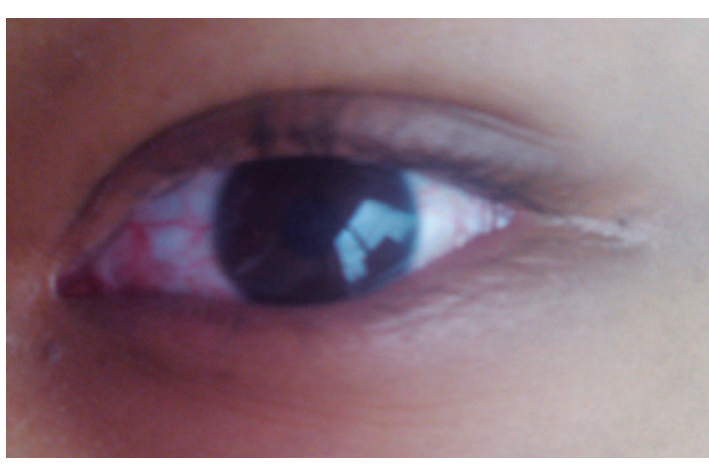

Figura 1. Telangiectasias oculares. 
ingreso a urgencias por dolor abdominal súbito e intenso (de predominio en mesogastrio), sin irradiación, sin exacerbantes ni atenuantes, sin fiebre; clínicamente se encontraron datos de abdomen agudo. Un ultrasonido abdominal mostró un tumor en el ovario derecho (Figura 2). Las concentraciones de alfafetoproteína y de fracción beta de la gonadotropina coriónica humana ( $\beta$-hGC) fueron $162 \mathrm{UI} / \mathrm{mL}$ y $>200 \mathrm{ng} / \mathrm{mL}$, respectivamente.

Se realizó laparotomía exploradora y se efectuó una salpingooforectomía derecha y apendicectomía. Se encontraron $400 \mathrm{~mL}$ de líquido peritoneal de aspecto hemorrágico y una masa tumoral sólida dependiente del ovario derecho, de aspecto blanquecino, con perforación en extremo superior y pedículo, que se extrajeron con facilidad.

El estudio anatomopatológico informó tumor ovárico multinodular de $13 \times 10 \times 7 \mathrm{~cm}$ con áreas sólidas de color blanco amarillento, zonas mixoides de aspecto necrótico y varios nódulos hemorrágicos. El nódulo mayor, de $4 \times 5 \mathrm{~cm}$, rodeaba y hacía cuerpo con la trompa uterina (Figuras 1 y 2). Histológicamente la neoplasia estaba constituida por células de núcleo redondo claro con nucléolo prominente. Células dispuestas en nidos, separadas por septos fibrosos con linfocitos (Figura 3). Las áreas hemorrágicas
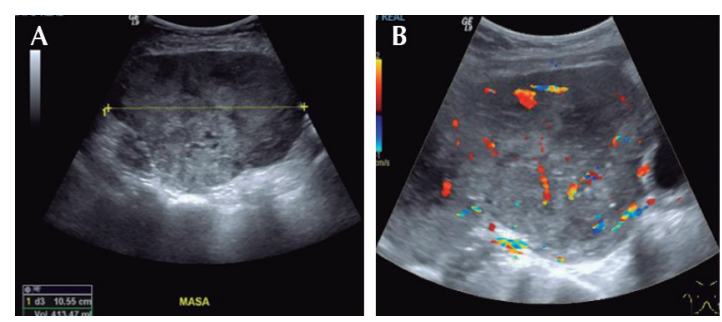

Figura 2. Ultrasonido en hueco pélvico. A) Masa sólida hipoecoica con zonas quísticas y ecos sugestivos de grasa, comprime superiormente la vejiga. B) Doppler color con vascularidad.

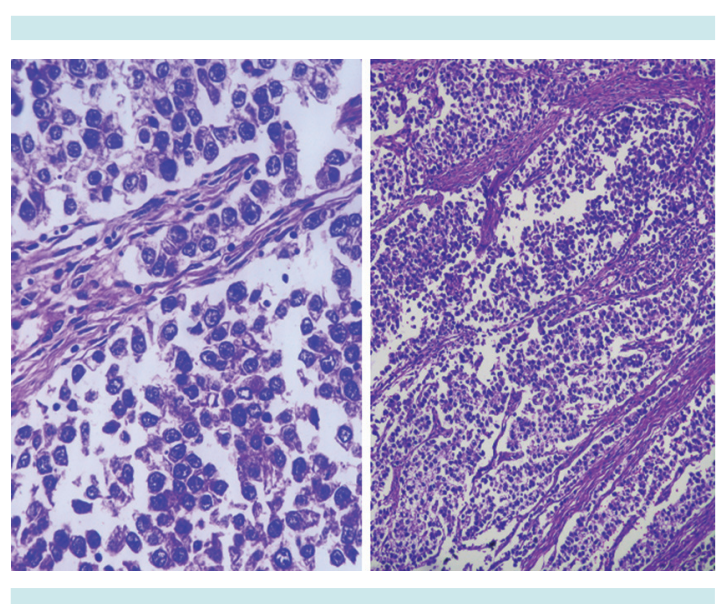

Figura 3. Áreas de disgerminoma con nidos de células de núcleo vesiculoso y nucléolo prominente separadas por septos fibrosos.

mostraron células de cito- y sincitiotrofoblasto (Figura 4).

Se identificó invasión vascular extensa e implantes de coriocarcinoma en el peritoneo y los tejidos blandos adyacentes. Con estos datos se estableció el diagnóstico de tumor maligno germinal mixto (con componente de disgerminoma y coriocarcinoma) con invasión vascular

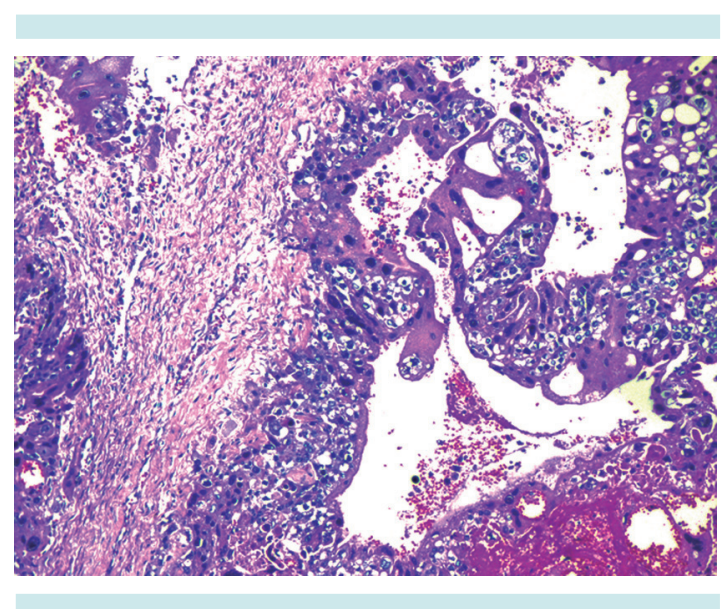

Figura 4. Áreas de coriocarcinoma con hemorragia y células de citotrofoblasto con células multinucleadas de sincitiotrofoblasto. 
al peritoneo y tejidos blandos perianexiales; se clasificó como estadio III de la Federación Internacional de Ginecología y Obstetricia (FIGO) para clasificación de tumores ováricos.

La evolución posquirúrgica fue satisfactoria. La alfafetoproteína disminuyó a $74 \mathrm{UI} / \mathrm{mL}$ y la fracción beta de la gonadotropina coriónica humana a 0 dos semanas después. Se inició ciclo de quimioterapia con bleomicina $\left(10 \mathrm{Ul} / \mathrm{m}^{2} /\right.$ día, dosis única), ciclofosfamida ( $1 \mathrm{~g} / \mathrm{m}^{2}$ por 4 días) y cisplatino $\left(20 \mathrm{mg} / \mathrm{m}^{2} /\right.$ día por cinco días). ${ }^{7} \mathrm{La}$ ciclofosfamida se usó en lugar del etopósido porque este medicamento mostró mayor riesgo de provocar segundas neoplasias y la susceptibilidad de la paciente por su diagnóstico de ataxia-telangiectasia. $^{7}$

La paciente presentó toxicidad hematológica grado IV según la clasificación del National Cancer Institute con choque séptico asociado con neumonía que requirió tratamiento en terapia intensiva, antibioticoterapia, apoyos ventilatorio y aminérgico. La recuperación hematológica de la paciente demoró más de seis semanas a pesar del factor estimulante de colonias de granulocitos y de transfusiones de hemoderivados en varias ocasiones.

Debido a que la paciente no tenía evidencia de actividad tumoral por imagen ni por marcadores bioquímicos, y la situación crítica en la que se encontró, se decidió dejarla en vigilancia sólo con un ciclo de quimioterapia. Actualmente se encuentra sin evidencia de actividad tumoral a 15 meses de seguimiento.

\section{DISCUSIÓN}

La ataxia-telangiectasia implica riesgo de 61 a 184 veces mayor que la población general de desarrollar neoplasias..$^{8,9}$ Este riesgo se incrementa por mutación de la proteína ATM que altera la función de reparación del ADN generando modificaciones del código genético y, secundariamente, activación de protooncogenes o genes supresores. Las principales neoplasias asociadas son: linfoma no Hodgkin $(40 \%)$, leucemias (25\%), enfermedad de Hodgkin (10\%) y tumores sólidos (25\%). De estos últimos se describen principalmente carcinoma gástrico, gonadoblastoma y meduloblastoma.

Su incidencia se estima de 1 por cada 40000 a 100000 nacidos vivos. Se acompaña de aumento de infecciones de las vías aéreas superiores en aproximadamente $80 \%$ de los pacientes. Las infecciones bacterianas, con menor frecuencia, pueden afectar otros sitios como piel, tejido óseo, tubo digestivo y genitouruinario. ${ }^{8}$

En 1991 Swift y sus colegas describieron a 161 familias de pacientes con ataxia-telangiectasia y su asociación con el cáncer de mama. Señalaron, por primera vez, la relación entre el estado heterocigoto para mutaciones del gen ATM con cáncer de mama, con una razón de oportunidad de 5.1 en mujeres para este tipo de cáncer, así como mayor mortalidad por todas las causas en adultos menores de 60 años, incrementado tres veces en hombres y 2.6 en mujeres. ${ }^{9}$ En la familia del caso que presentamos no se refirieron casos previos de cáncer.

No existe tratamiento específico para la ataxiatelangiectasia. ${ }^{10}$ Los pacientes son atendidos con terapias de rehabilitación, suplementos con vitamina $B_{12}$ o vitamina $E$ pero sin resultados concluyentes. ${ }^{11}$ Los corticosteroides, en especial la betametasona en dosis baja $(0.1 \mathrm{mg} /$ $\mathrm{kg}$ /día por cuatro semanas), se han usado para tratar la ataxia pero los resultados a corto plazo no son satisfactorios. ${ }^{10,11}$ En lo inmunológico se recomienda la terapia con inmunoglobulina humana intravenosa de reemplazo y profilaxis antimicrobiana, así como tratamiento oportuno de infecciones. ${ }^{11}$ También se recomienda el asesoramiento genético dado el riesgo de 
recurrencia de $25 \%$ en otros hijos con ataxiatelangiectasia.

El seguimiento multidisciplinario y el tratamiento integral de los pacientes con ataxia-telangiectasia incluye vigilancia activa y periódica por parte de oncología. No existe un consenso general respecto a qué estudios y con qué frecuencia se deben realizar. Diferentes autores proponen seguimiento semestral con biometrías hemáticas y ultrasonidos abdominales en búsqueda de leucemias y linfomas abdominales, respectivamente. La utilidad de los marcadores como la alfafetoproteína y la fracción beta de la gonadotropina coriónica humana no ha sido evaluada apropiadamente y más de $95 \%$ de los pacientes con ataxia-telangiectasia tienen niveles de alfafetoproteína $>10 \mathrm{mg} / \mathrm{dL}$, independientemente de la existencia de neoplasia. La presentación clínica de las leucemias y linfomas suele ser similar a otros pacientes sin ataxia-telangiectasia. Por otro lado, la presentación más frecuente de los tumores ováricos (coriocarcinoma y disgerminoma) son: abdomen agudo (80\%), dolor abdominal crónico (10\%), aumento de volumen (5\%), síntomas de pubertad precoz (5\%). ${ }^{12}$

Realizamos una revisión de la literatura en PubMed, Artemisa y Embase, utilizando como palabras clave los términos MeSH (Medical Subject Headings): ovaric neoplasia, ovaric cancer, dysgerminoma, coriocarcinoma, leukemia, adolescent y ataxia-telangiectasia; identificamos siete artículos de pacientes con ataxia-telangiectasia y tumores de ovario.

En 1964 Dunn y su equipo describieron el caso de una paciente de 17 años con ataxia-telangiectasia y diagnóstico (por autopsia) de tumor de ovario. ${ }^{13}$ En 1975 Goldsmith y su grupo describieron un caso similar, de una adolescente también de 17 años, con diagnóstico de ataxia-telangiectasia a los 13 años, con gonadoblastoma del ovario izquierdo y disgerminoma contralateral, pro- pusieron la relación de dichas neoplasias con la ataxia-telangiectasia. ${ }^{14}$ Un año más tarde, Byuse y sus colegas describieron un caso en una paciente de las mismas edad y características. ${ }^{15}$ En 1984 Narita y Tagaki describieron el caso de una paciente con ataxia-telangiectasia y múltiples neoplasias, entre ellas disgerminoma de ovario. ${ }^{16}$ En 1988 Pecorelli y sus colaboradores publicaron el primer caso de ataxia-telangiectasia y tumor de ovario de senos endodérmicos, tratada con cirugía y dos ciclos de quimioterapia con cisplatino, vinblastina y bleomicina, que sobrevivió pero sin especificar el tiempo de seguimiento. ${ }^{17}$ En 1997 DeVries y su grupo describieron el caso de una adolescente de 16 años con ataxia-telangiectasia que sufría incontinencia urinaria, se le diagnosticó metástasis de disgerminoma a vejiga urinaria; fue tratada con quimioterapia intensa y no hubo evidencia de la enfermedad en 24 meses de seguimiento. ${ }^{18}$ En 2007 Koksal y sus colegas describieron una paciente con ataxia-telangiectasia, aumento progresivo de alfafetoproteína, antígeno de cáncer 125 (Ca 125) y de la fracción beta de la gonadotropina coriónica humana: se le diagnosticó disgerminoma de ovario ${ }^{19}$ y establecieron la importancia de la vigilancia activa de neoplasias en el seguimiento de los adolescentes con ataxiatelangiectasia (Cuadro 1).

Hay que destacar el dilema ético que existe en estos pacientes. Hay protocolos establecidos, en cuanto a dosis y número de ciclos de quimioterapia, para el tratamiento de distintas neoplasias pero para pacientes sin otra afección concomitante con el cáncer. Pero existen situaciones especiales como las de las pacientes con ataxia-telangiectasia donde la reparación del ADN está comprometida y esto las hace más susceptibles a los efectos citotóxicos de los agentes antineoplásicos; se incrementa también el riesgo de complicaciones y del desarrollo de segundas neoplasias. Por estas razones se realizan modificaciones a los protocolos de tratamiento en este tipo de pacientes. 
Cuadro 1. Casos de ataxia-telangiectasia y cáncer de ovario consultados en PubMed, Artemisa y Embase a diciembre del 2013

\begin{tabular}{|c|c|c|c|c|c|c|}
\hline Referencia & Tipo de neoplasia & Topografía & $\begin{array}{l}\text { Edad de } \\
\text { diagnóstico } \\
\text { de AT* }\end{array}$ & $\begin{array}{c}\text { Edad de } \\
\text { diagnóstico } \\
\text { del tumor }\end{array}$ & Tratamiento & Desenlace \\
\hline Dunn, $1964^{13}$ & Tumor de ovario & Bilateral & 13 años & 17 años & $\begin{array}{l}\text { Ninguno Diagnós- } \\
\text { tico por autopsia }\end{array}$ & $\begin{array}{l}\text { Falleció por neu- } \\
\text { monía }\end{array}$ \\
\hline $\begin{array}{l}\text { Goldsmith } \\
1975^{14}\end{array}$ & $\begin{array}{l}\text { Gonadoblastoma y } \\
\text { disgerminoma }\end{array}$ & Derecho & 13 años & 17 años & Cirugía & $\begin{array}{l}\text { Falleció por neu- } \\
\text { monía }\end{array}$ \\
\hline $\begin{array}{l}\text { Byuse } \\
1976^{15}\end{array}$ & Disgerminoma & Izquierdo & No refiere & 17 años & Cirugía & $\begin{array}{l}\text { Falleció a los } \\
\text { cuatro meses por } \\
\text { neumonía }\end{array}$ \\
\hline $\begin{array}{l}\text { Narita } \\
1984^{16}\end{array}$ & $\begin{array}{l}\text { Disgerminoma de } \\
\text { ovario derecho, car- } \\
\text { cinoma papilar de } \\
\text { tiroides y adenocar- } \\
\text { cinoma de páncreas }\end{array}$ & Derecho & No refiere & 19 años & $\begin{array}{l}\text { Cirugía } \\
\text { Radioterapia }\end{array}$ & $\begin{array}{l}\text { Falleció por neu- } \\
\text { monía }\end{array}$ \\
\hline $\begin{array}{l}\text { Pecorelli } \\
1988^{17}\end{array}$ & $\begin{array}{l}\text { Tumor de senos, } \\
\text { endodérmico de } \\
\text { ovario }\end{array}$ & Izquierdo & 6 años & 15 años & $\begin{array}{l}\text { Cirugía } \\
\text { Quimioterapia: } \\
\text { cisplatino ( } 20 \mathrm{mg} / \\
\mathrm{m}^{2} / \mathrm{dia} \text { ) por } 3 \\
\text { días, vinblastina } \\
\text { ( } 0.15 \mathrm{mg} / \mathrm{kg} / \mathrm{día}) \\
\text { por dos días y bleo- } \\
\text { micina ( } 30 \mathrm{mg} \text { en } \\
\text { infusión por } 24 \text { h). } \\
\text { El segundo ciclo } \\
\text { se administró a la } \\
\text { mitad de la dosis } \\
\text { de cada uno }\end{array}$ & $\begin{array}{l}\text { Sobrevivió. No } \\
\text { menciona tiempo }\end{array}$ \\
\hline $\begin{array}{l}\text { Devries } \\
1997^{18}\end{array}$ & $\begin{array}{l}\text { Disgerminoma con } \\
\text { metástasis vesicu- } \\
\text { lares }\end{array}$ & Izquierdo & No refiere & 16 años & $\begin{array}{l}\text { Cirugía } \\
\text { Quimioterapia (cis- } \\
\text { platino y vinblasti- } \\
\text { na) dos ciclos. No } \\
\text { menciona dosis }\end{array}$ & $\begin{array}{l}\text { Sobrevivió a dos } \\
\text { años de } \\
\text { seguimiento }\end{array}$ \\
\hline $\begin{array}{l}\text { Koksal } \\
2007^{19}\end{array}$ & $\begin{array}{l}\text { Disgerminoma no } \\
\text { metastásico }\end{array}$ & Derecho & 1 año & 10 años & $\begin{array}{l}\text { Cirugía } \\
\text { Carboplatino } \\
\text { ( } 450 \mathrm{mg} / \mathrm{m}^{2} / \text { día } \\
\text { un día), etopósi- } \\
\text { do ( } 100 \mathrm{mg} / \mathrm{m}^{2} / \\
\text { día, por tres días) } \\
\text { bleomicina ( } 10 \mathrm{mg} / \\
\mathrm{m}^{2} / \text { día, por dos } \\
\text { días) Dos ciclos de } \\
\text { quimioterapia }\end{array}$ & $\begin{array}{l}\text { Sobrevivió a dos } \\
\text { años de } \\
\text { seguimiento }\end{array}$ \\
\hline
\end{tabular}

*AT: ataxia-telangiectasia.

En nuestro caso se hizo la resección quirúrgica del tumor y se inició la quimioterapia con ciclofosfamida, bleomicina y cisplatino. Se modificó el protocolo estándar propuesto por
Pizzo y Poplack, en 2010, y se decidió sustituir el etopósido por ciclofosfamida debido a la asociación del primero con segundas neoplasias. ${ }^{20}$ Se administraron las dosis recomendadas aunque 
sólo se administró un ciclo de quimioterapia (de los cuatro recomendados) debido a la toxicidad elevada que tuvo en la paciente; ${ }^{20}$ actualmente continúa en vigilancia estrecha sin evidencia de actividad tumoral a 17 meses de seguimiento.

Se propone, asimismo, un seguimiento preventivo más estrecho a este tipo de pacientes a partir del diagnóstico de ataxia-telangiectasia. Además de los estudios establecidos para la búsqueda de leucemias y linfomas se deben realizar marcadores séricos tumorales (alfafetoproteína, fracción beta de la gonadotropina coriónica humana) y ultrasonido abdominal cada cuatro a seis meses. También se debe insistir en evitar radiación innecesaria y, lo más importante, la exploración física cuidadosa enfocada a la detección oportuna del cáncer. Al tener un diagnóstico oportuno estaremos en posibilidad de ofrecer un tratamiento de menor intensidad y con ello disminuir posibles complicaciones. En este caso mostramos un esquema de quimioterapia menos agresivo en tiempo y número de ciclos, sumado a resección quirúrgica previa, con buenos resultados hasta el momento.

\section{CONCLUSIÓN}

Este es un caso clínico poco frecuente. Es necesario proponer una estrategia de seguimiento y de tratamiento oncológico en este tipo de pacientes. La alfafetoproteína no es útil en estos pacientes tomando sólo una determinación, pero las elevaciones por encima de lo esperado, o aumentos abruptos de concentraciones séricas justificarían la extensión de los estudios con miras a determinar patología neoplásica. Estos casos nos enfrentan con dilemas éticos en los que probablemente debamos establecer conductas particulares para el tratamiento del cáncer. En este caso establecimos un tratamiento multidisciplinario por parte de los servicios de oncología médica, urgencias, inmunología, cirugía oncológica, patología, psicología y trabajo social que benefició a nuestra paciente. La dosis de quimioterapia fue menor a la establecida en los protocolos estándar, lo que permite preguntarse acerca de la necesidad de estratificar un grupo especial de pacientes con inmunodeficiencias y cáncer.

\section{REFERENCIAS}

1. Lavin MF, Gueven N, Bottle S, Gatti RA. Current and potential therapeutic strategies for the treatment of ataxiatelangiectasia. Queensland Institute of medical Research, Brisbane. Australia Br Med Bull 2007;81:129-47.

2. Frappart PO, and McKinnon PJ. Ataxia-telangiectasia and related diseases. Department of genetics and tumour biology. St Jude Childrens Research Hospital. Memphis. Rev Neuromolecular 2006;8:495:511.

3. Ambrose M, Gatti R. Pathogenesis of ataxia-telangiectasia: the next generation ATM functions. University of Tasmania. Australia. Department of molecular biology. UCLA school of Medicine. Los Angeles, CA. Rev Blood 2013;121:4036-4045.

4. Kishi S. Zho Xz, Ziv Y, khoo C, Hill DE, et al. Telomeric protein Pin2/TRF1 as un important ATM target in response to double stran DNA breaks. J Boil Chem 2011;276(20):16587-93.

5. Li N, Banin S, Ouyang H, Li GC, courtis G et al. ATM is required for Ikappa $B$ kinase activation in reponse to DNA doauble strand breaks. J Biol Chen 2001;276(12):898-903.

6. 6Kastan M. Ataxia telangiectasia gene linked to insulin function. Nat Cell Biol 200;(12):893-8.

7. Spacey SD, Gatti RA, Bebb G. The molecular basis and clinical management of ataxia telangiectasia. Can J Neurol Sci 2000;27(3):184-91.

8. FIGO Committee on Gynecologic Oncology: Current FIGO staging for cancer of the vagina, fallopian tube, ovary, and gestational trophoblastic neoplasia. Int J Gynaecol Obstet 2009;105(1):3-4.

9. Swift M, Morrell D, Massey RB, Chase CL. Incidence of cancer in 161 families affected by ataxia-telangiectasia. University of North Carolina. The New England Journal of Medicine 1991;325:1831-6.

10. Sabrina Buoni, Raffaella Zannolli, Livio Sorrentino, Alberto Fois. Betamethasone and Improvement of Neurological. Symptoms in Ataxia-Telangiectasia. University of Siena. Italia. Arch Neurol 2006;63:1480.

11. Anheim M, Tranchant $C$, Koenig M. The autosomal recessive cerebellar ataxias Universidad Pierre et Marie Curie. Paris. The New England Journal of Medicine 2012;366:636-46.

12. Gatti R, Pagon RA, Bird TD, Dolan CR, Stephens K, Adam MP. Ataxia-Telangiectasia. Department of Pathology. School of Medicine. UCLA California. GeneReviews. http://www. ncbi.nlm.nih.gov/pubmed/20301790 
13. Dunn HG, Meuwisssen H, Livingstone CS, Pump K. Ataxiatelangiectasia. Can Med Assoc J 1964;91:1106-18.

14. Goldsmith $\mathrm{Cl}$, Hart WR. Ataxia-telangiectasia with ovarian gonadoblastoma and contralateral dysgerminoma. Cancer 1975;36(5):1838-42.

15. Buyse M, Hartmen CT, Wilson MG. Gonadoblastoma and dysgerminoma with ataxia-telangiectasia. Birth Defects Orig Artic Ser 1976;12(1):165-9.

16. Narita T, Takagi K. Ataxia - telangiectasia with dysgerminoma of right ovary, papillary carcinoma of thyroid, and adenocarcinoma of pancreas. Cancer 1984;54(6):1113-6.

17. Pecorelli S, Sartori E, et al. Ataxia-telangiectasia and endodermal sinus tumor of the ovary: report of a case. Gynecologic. Oncology 1998;29(2):240-4.
18. DeVries CR, Kaplan GW. An unusual case of urinary incontinence, ataxia-telangiectasia, and metastatic dysgerminoma: case report and review of the literature Urology1997;50(3):453-5.

19. Koksal Y, Caliskan U, Ucar C, Yurtcu M, Artac H, llerisoyYakut Z, Reisli Dysgerminoma in a child with ataxiatelangiectasia. Pediatr Hematol Oncol 2007;24(6):431-6.

20. Pizzo P, Poplack D. Principles and Practice of Pediatric Oncology. 6th edition. EU, Germ Cell Tumors. Wolters Kluwers. 2010 pp. 1045-1067. 